\title{
Frequency-dependent attenuation of $S$-waves in the Kanto region, Japan
}

\author{
Kazuo Yoshimoto and Mariko Okada \\ International Graduate School of Arts and Sciences, Yokohama City University, 22-2 Seto, Kanazawa, Yokohama 236-0027, Japan
}

(Received December 18, 2008; Revised March 27, 2009; Accepted June 2, 2009; Online published November 10, 2009)

\begin{abstract}
Apparent, scattering, and intrinsic $S$-wave attenuations $\left(Q_{S}^{-1}, Q_{\text {scat }}^{-1}\right.$ and $\left.Q_{\text {int }}^{-1}\right)$ of the upper lithosphere in the Kanto region of Japan were measured in the $1-$ to $32-\mathrm{Hz}$ frequency range using Multiple Lapse Time Window Analysis (MLTWA) for 115 borehole seismograms of local earthquakes. A new set of time windows for MLTWA, in which multiple isotropic scattering is assumed, was proposed and employed to estimate the frequency dependence of $S$-wave attenuation parameters. Scattering attenuation was found to dominate intrinsic attenuation in the $S$-wave attenuation mechanism at low frequencies $(<2 \mathrm{~Hz})$, whereas the opposite relation was observed at high frequencies. The transition is caused by the different frequency dependences of $Q_{\text {scat }}^{-1}\left(\propto f^{-1.5}\right)$ and $Q_{\text {int }}^{-1}\left(\propto f^{-0.7}\right)$ at this frequency. Interestingly, $Q_{\text {scat }}^{-1}$ is almost frequency independent at frequencies $>8 \mathrm{~Hz}$, which implies the self-similar nature of short-wavelength heterogeneities in the upper lithosphere. In terms of the upper lithosphere of the Kanto region, these results may indicate that the random heterogeneities characterized by the Gaussian autocorrelation function with a fractional fluctuation $\varepsilon \approx 10 \%$ and a correlation length $a \approx 2 \mathrm{~km}$ are superimposed on the weak background self-similar heterogeneity.
\end{abstract}

Key words: $S$-wave attenuation, scattering attenuation, intrinsic attenuation, heterogeneities in the upper lithosphere, Multiple Lapse Time Window Analysis.

\section{Introduction}

The Kanto region of Honshu Island, which is the most densely populated metropolitan area of Japan, has high seismicity associated with the subductions of the Philippine Sea Plate and the Pacific Plate beneath the North American Plate. To improve the accuracy of strong ground motion prediction and prevent the seismic hazards that will be induced by large earthquakes, we have to obtain precise information on the underground structure and seismic wave attenuation in the crust and upper mantle (the upper lithosphere).

The apparent attenuation of $S$-waves in the upper lithosphere has been studied in and around this region using spectral inversion techniques with strong motion data accumulated from array observations (Takemura et al., 1991; Kinoshita, 1994; Kato et al., 1998). The coda normalization method, which can be applied to single-station data from local earthquakes with widespread hypocenters, is another major technique for estimating the apparent attenuation of $S$-waves (e.g., Aki, 1980a, b; Yoshimoto et al., 1993). Previous studies of $S$-wave attenuation in this region have revealed that $Q_{S}^{-1}$ exhibits clear frequency dependence and that it decreases with increasing frequency at frequencies of $1-10 \mathrm{~Hz}$, with $Q_{S}^{-1} \approx 1 \times 10^{-2}$ at $1 \mathrm{~Hz}$ and $Q_{S}^{-1} \approx 2 \times 10^{-3}$ at $10 \mathrm{~Hz}$. Despite the fact that many reliable measurements have been performed in relation to the frequency dependence of $Q_{S}^{-1}$, there have been few studies that separately estimate the quality factors for scattering and intrinsic at-

Copyright (c) The Society of Geomagnetism and Earth, Planetary and Space Sciences (SGEPSS); The Seismological Society of Japan; The Volcanological Society of Japan; The Geodetic Society of Japan; The Japanese Society for Planetary Sciences; TERRAPUB tenuations (Fehler et al., 1992; Hoshiba, 1993).

In this study, we estimate the apparent, scattering, and intrinsic attenuations of $S$-waves $\left(Q_{S}^{-1}, Q_{\text {scat }}^{-1}\right.$ and $\left.Q_{\text {int }}^{-1}\right)$ in the upper lithosphere beneath the Kanto region in the 1- to 32-Hz frequency range using Multiple Lapse Time Window Analysis (MLTWA). We propose a new set of time windows for MLTWA in which multiple isotropic scattering is assumed and employ this set to measure the frequency dependence of the attenuation parameters of $S$-waves in detail. We also propose a stochastic structure model that characterizes the random heterogeneities in the upper lithosphere of the Kanto region.

\section{Data}

The Edosaki station $\left(35.9521^{\circ} \mathrm{N}, 140.3301^{\circ} \mathrm{E}\right)$, which is one of the borehole observation stations that constitute the Japanese high-sensitivity seismograph network (Hi-net: Okada et al., 2004) operated by the National Research Institute for Earth Science and Disaster Prevention, is located about $60 \mathrm{~km}$ northeast of Tokyo (Fig. 1). This station is in an area where the seismic basement is not in the deep ground and situated far from the active volcanoes surrounding the Kanto Plain. The borehole penetrates $660 \mathrm{~m}$ into sedimentary layers and then into basement rock to reach a total depth of $1,230 \mathrm{~m}$. A three-component velocity seismometer with a natural frequency of $1 \mathrm{~Hz}$ is installed $1,200 \mathrm{~m}$ below the surface. Output signals from this seismometer are digitized with 24-bit resolution at $100-\mathrm{Hz}$ sampling.

The data set used in this study consists of 115 seismograms from local earthquakes recorded between April 2003 and December 2007 (Fig. 1). The magnitudes and focal 


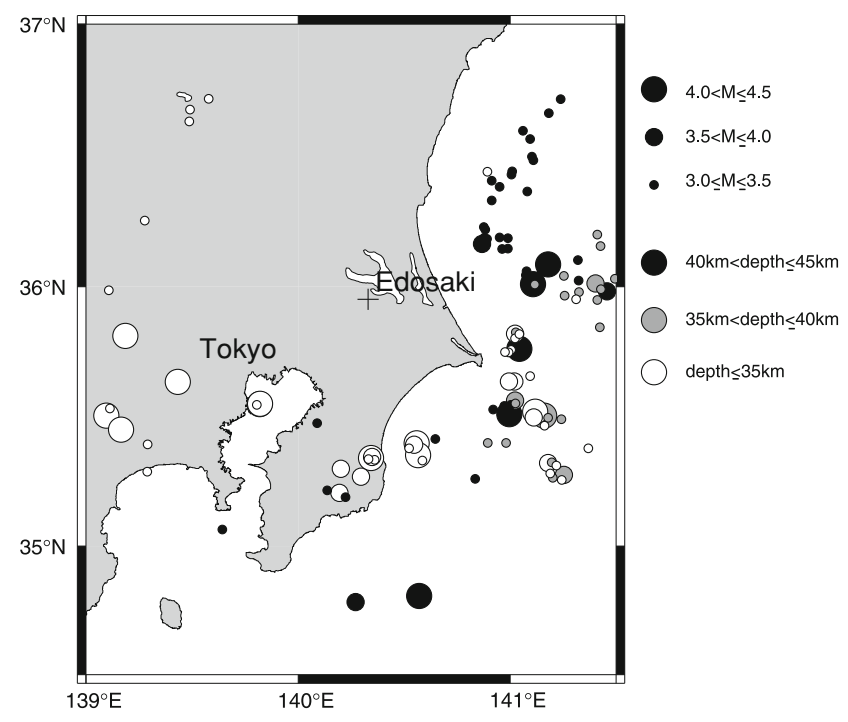

Fig. 1. Map showing the location of the seismic station and the 115 local earthquakes used in this study.

depths of these events are in the ranges of 3.0-4.5 and 0$45 \mathrm{~km}$, respectively. The hypocentral distance calculated from the hypocenter parameter list provided by the Japan Meteorological Agency (Ueno et al., 2002) ranges from 70 to $140 \mathrm{~km}$. This hypocentral range ensures a sufficient number of local earthquakes for the analysis to estimate the attenuation parameters of $S$-waves.

\section{Envelope Analysis}

\subsection{Multiple isotropic scattering model}

The multiple isotropic scattering model (Zeng et al., 1991) was adopted to synthesize theoretical envelopes for MLTWA. Zeng et al. (1991) derived an integral equation that expresses the energy density of seismic waves for a given location $\mathbf{r}$ and lapse time $t$ resulting from an impulsive point source located at the origin:

$$
\begin{aligned}
E(\mathbf{r}, f \mid t)= & \frac{W e^{-L_{\mathrm{e}}^{-1}|\mathbf{r}|}}{4 \pi V_{S}|\mathbf{r}|^{2}} \delta\left(t-\frac{|\mathbf{r}|}{V_{S}}\right) \\
& +L_{\mathrm{e}}^{-1} B_{0} \int_{-\infty}^{\infty} \int_{-\infty}^{\infty} \int_{-\infty}^{\infty} \int_{-\infty}^{\infty} E\left(\mathbf{r}^{\prime}, f \mid t^{\prime}\right) \frac{e^{-L_{\mathrm{e}}^{-1}\left|\mathbf{r}-\mathbf{r}^{\prime}\right|}}{4 \pi\left|\mathbf{r}-\mathbf{r}^{\prime}\right|^{2}} \\
& \cdot \delta\left(t-t^{\prime}-\frac{\left|\mathbf{r}-\mathbf{r}^{\prime}\right|}{V_{S}}\right) d t^{\prime} d \mathbf{r}^{\prime}
\end{aligned}
$$

where $f$ is the frequency, $V_{S}$ is the average $S$-wave velocity, and $\delta(t)$ is the Dirac delta function. The seismic energy density is parameterized in terms of the seismic albedo $B_{0}\left(=Q_{\text {scat }}^{-1} /\left(Q_{\text {scat }}^{-1}+Q_{\text {int }}^{-1}\right)\right)$ and the inverse of the extinction length $L_{\mathrm{e}}^{-1}\left(=2 \pi f\left(Q_{\text {scat }}^{-1}+Q_{\text {int }}^{-1}\right) / V_{S}\right)$. The first term on the right side of Eq. (1) represents the propagation of direct wave energy from the source to the receiver, while the second term is the sum of the scattered energy from all possible scatterers located at $\mathbf{r}^{\prime}$. This integral equation can be solved numerically in the integral transform domain by using the FFT algorithm. Rather than assuming that the earth has a very heterogeneous structure, this model assumes a uniform scatterer distribution in an unbounded uniform background medium. However, all multiple scatterings can be evaluated rigorously. Here we refer to $E(\mathbf{r}, f \mid t)$, which is used in MLTWA, as the theoretical seismic energy.

\subsection{Multiple Lapse Time Window Analysis}

To estimate the scattering and intrinsic attenuations of the high-frequency $S$-waves in the upper lithosphere separately, the MLTWA technique was applied to the seismogram envelopes of 115 events. To achieve a frequency analysis, NScomponent seismograms were bandpassed using 4-pole and $1 / 2$ octave pass-band Butterworth filters for 19 frequency bands ranging between 1 and $32 \mathrm{~Hz}$ (Fig. 2). The envelopes were then evaluated taking the mean square of these filtered traces.

In conventional MLTWA, three consecutive time windows of the same length from an $S$-wave onset are used to investigate the temporal variation in seismic energy. It is natural to expect an estimation obtained using a single time window to be consistent with that obtained using conventional three time-window analysis and other attenuation measurement techniques (e.g., the coda normalization method). However, our MLTWA analysis only using the first time window, whose length is longer than $10 \mathrm{~s}$, underestimated $Q_{S}^{-1}$ significantly compared with the value obtained with the coda normalization method. This may be explained in terms of an incorrect theoretical prediction of early coda energy from the multiple isotropic scattering model (Hoshiba, 1995). To remove this inconsistency, the length of the first time window should be sufficiently short so as to cover only the direct $S$-waves by excluding the early coda waves from this time window. Consequently, we adjusted the length of the first time window of our MLTWA to $5 \mathrm{~s}$, as used in the coda normalization method for $Q_{S}^{-1}$ estimation reported by Yoshimoto et al. (1993). This time length roughly corresponds to the expected maximum duration of the principal $S$-wave motion, which is formed by the "broadening" that occurs due to the effects of wave propagation through the heterogeneous upper lithosphere (Saito et al., 2005).

The second and third time windows of the MLTWA contribute to the separate estimation of $Q_{\text {scat }}^{-1}$ and $Q_{\text {int }}^{-1}$. Despite these problems discussed above, the multiple isotropic scattering model accurately predicts temporal variations in seismic energy, which are mainly characterized by $Q_{\text {int }}^{-1}$, when the lapse time increases sufficiently. Therefore, in this analysis, the second and third time windows were set at lapse times of 60-65 and 70-75 s, respectively.

Unlike the uniform coda energy distribution observed for local earthquakes (Tsumura, 1967), the multiple isotropic scattering model predicts a concentration of seismic energy around the hypocenter even for large lapse times. To eliminate this hypocentral dependence, in our MLTWA, the coda normalization for theoretical seismic energy in the first time window was achieved by using its value at a lapse time of 80-85 s and a reference hypocentral distance of $100 \mathrm{~km}$ (midpoint value of hypocentral range) irrespective of the earthquake. Study showed that our results are not sensitive to these values (the maximum variations fall within the estimated errors).

By using the grid search analysis technique proposed by Hoshiba (1993), the observed data was compared with 

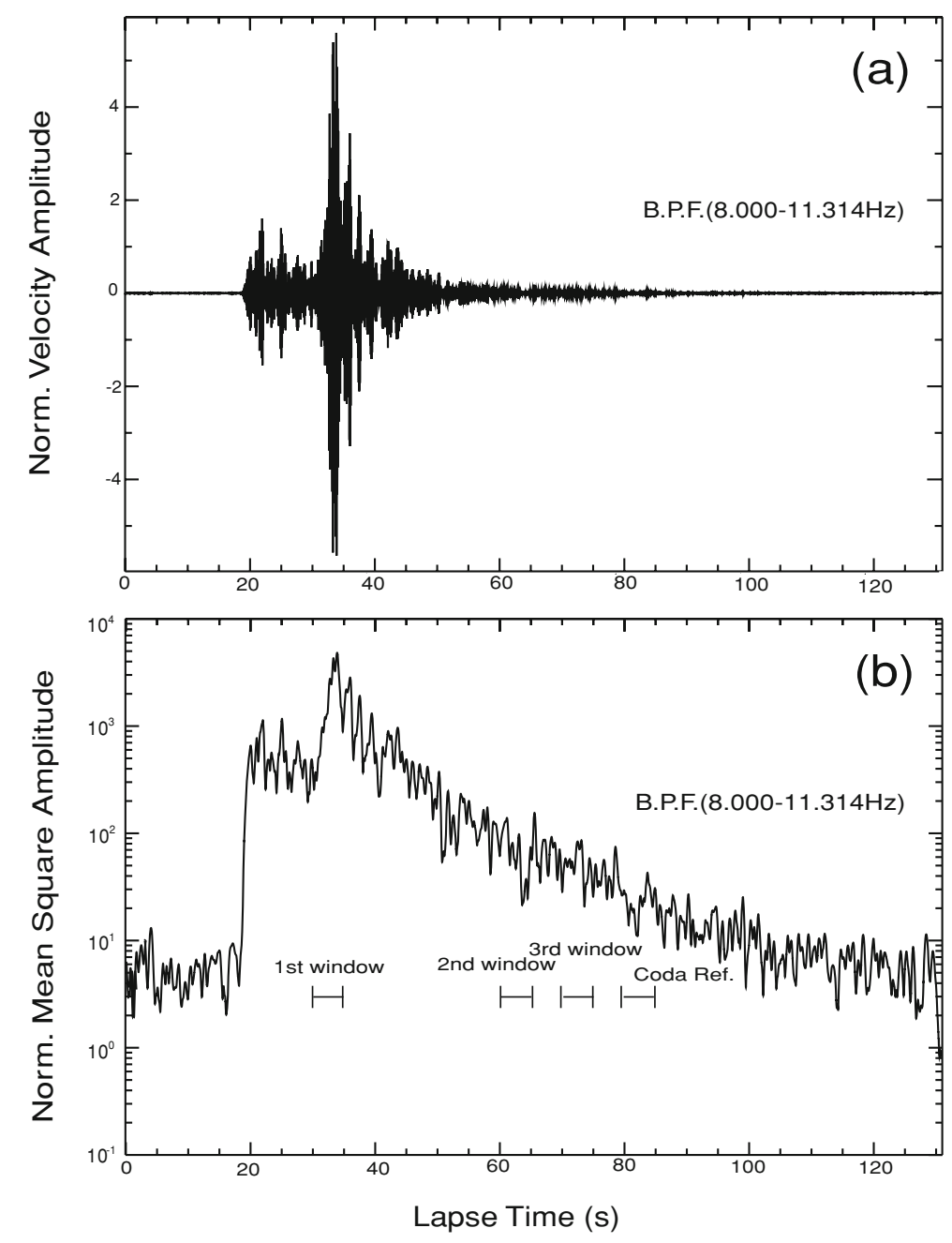

Fig. 2. (a) Example of a band-pass-filtered seismogram. (b) Corresponding example of a mean square envelope. Three time windows used for seismic energy estimation and the coda reference window for MLTWA are shown below the envelope.

model predictions from Eq. (1) to estimate the best model parameters for explaining the spatio-temporal distribution of the seismic energy of local earthquakes (Fig. 3). This analysis was carried out at 6106 grid points that cover an appropriate range of variability for two model parameters: $L_{\mathrm{e}}^{-1}=0.005,0.006,0.007, \cdots, 0.090 \mathrm{~km}^{-1}$ and $B_{0}=0.10,0.11,0.12, \cdots, 0.80$. Using an average $S$-wave velocity of $4 \mathrm{~km} / \mathrm{s}$, a set of $S$-wave attenuation parameters for the upper lithosphere can be obtained from the following relations: $Q_{S}^{-1}=L_{\mathrm{e}}^{-1} \cdot V_{S} / 2 \pi f, Q_{\text {scat }}^{-1}=B_{0} \cdot Q_{S}^{-1}$, and $Q_{\text {int }}^{-1}=\left(1-B_{0}\right) \cdot Q_{S}^{-1}$.

Figure 4 shows the distribution of the residuals between the observed and model predicted data in the model parameter space. The grid point corresponding to the minimum residual is indicated by a solid circle. To estimate the error intervals in $L_{\mathrm{e}}^{-1}$ and $B_{0}$, we used the $F$ distribution at a $68 \%$ level of confidence (Abubakirov, 2005). Figure 3 shows the model prediction for the best fitting $L_{\mathrm{e}}^{-1}$ and $B_{0}$. The theoretical prediction well explains the hypocentral variation in the observed data, except for that from the first time window in which the source radiation pattern and/or the focusing and defocusing of direct $S$-waves (Hoshiba, 2000) might have a strong effect.

\section{Results}

The values of the estimated attenuation parameters $L_{\mathrm{e}}^{-1}$, $B_{0}, Q_{S}^{-1}, Q_{\text {scat }}^{-1}$, and $Q_{\text {int }}^{-1}$ for each frequency band are listed in Table 1. Figure 5 shows the $Q_{S}^{-1}$ values obtained in this study, together with those obtained by other studies undertaken in and around the Kanto region for comparison. Our estimations agree reasonably well with those of other studies as regards absolute value and frequency dependence, and confirm that, in the Kanto region, $Q_{S}^{-1}$ shows a clear frequency dependence, decreasing with increasing frequency at frequencies of $1-10 \mathrm{~Hz}: Q_{S}^{-1} \approx 1 \times 10^{-2}$ at $1 \mathrm{~Hz}$ and $Q_{S}^{-1} \approx 2 \times 10^{-3}$ at $10 \mathrm{~Hz}$. The consistency among different observations implies that our estimation is stable as the spatial average of the variation in $Q_{S}^{-1}$ in this region (e.g., Nakamura et al., 2006). It is interesting to note that the $Q_{S}^{-1}$ decay rate with increasing frequency is not stationary but changes at approximately 2 and $8 \mathrm{~Hz}$.

Figure 6 shows the frequency dependence of $Q_{S}^{-1}, Q_{\text {scat }}^{-1}$ and $Q_{\text {int }}^{-1}$ obtained in this study. This figure indicates that the scattering is a predominant attenuation mechanism $\left(B_{0}>\right.$ $0.5)$ at frequencies $<2 \mathrm{~Hz}$, whereas intrinsic attenuation is dominant over scattering attenuation $\left(B_{0}<0.5\right)$ at high frequencies. It is worth noting that the change in the frequency dependence of $Q_{S}^{-1}$ observed at a frequency of $2 \mathrm{~Hz}$ can 

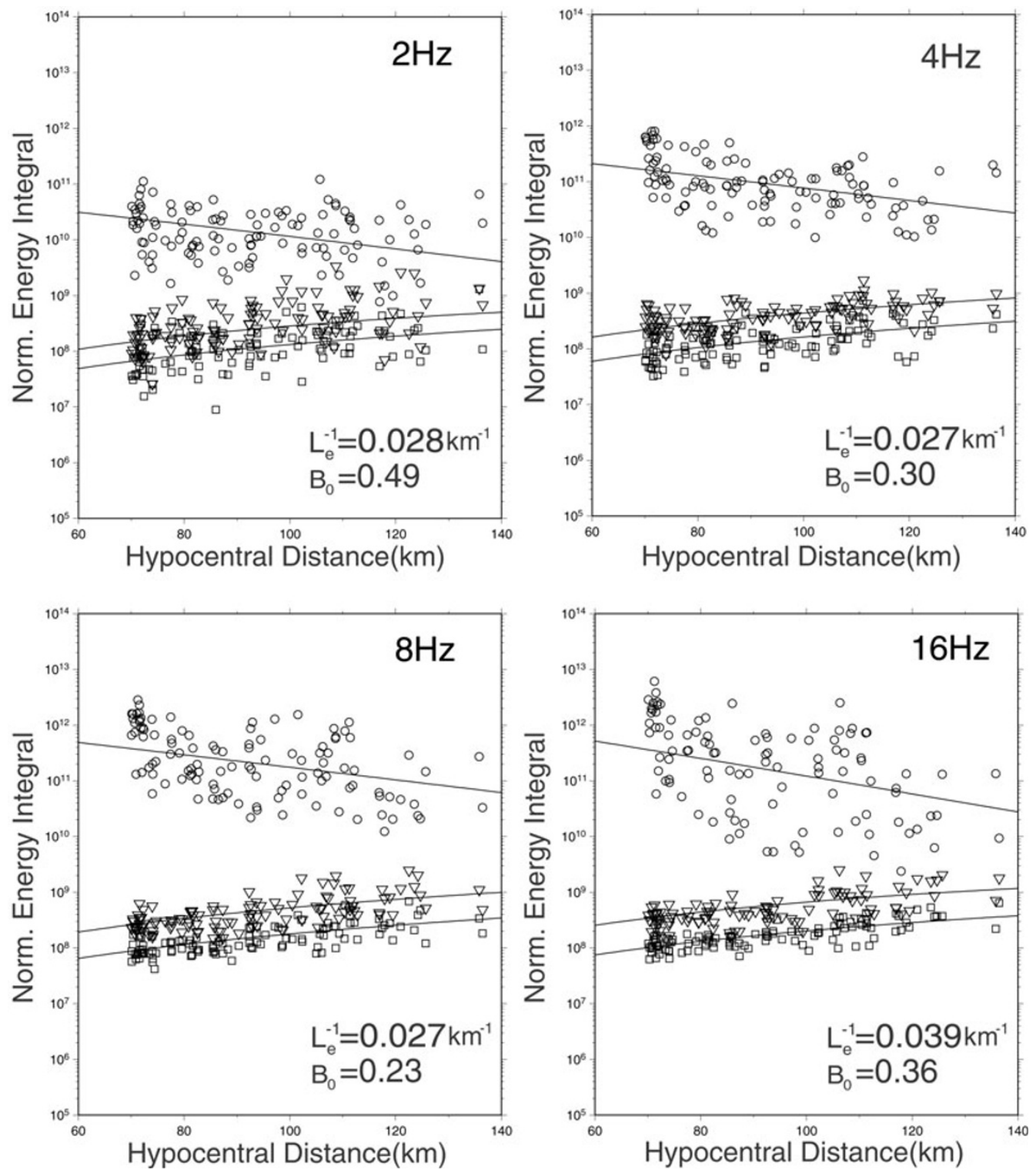

Fig. 3. MLTWA results from four frequency bands (center frequencies of 2, 4, 8, and $16 \mathrm{~Hz}$ ). Best fitting model curves from estimated $L_{\mathrm{e}}^{-1}$ and $B_{0}$ are indicated by solid lines. Circles, inverted triangles, and squares represent observed data from the first, second, and third time windows, respectively.

be interpreted by the transition of the dominant attenuation mechanism. It is observed that $Q_{\text {scat }}^{-1}$ decreases in proportion to $f^{-1.5}$ at frequencies below about $8 \mathrm{~Hz}$. However, $Q_{\text {scat }}^{-1}$ becomes almost frequency independent at high frequencies. This characteristic introduces the second change in the frequency dependence of $Q_{S}^{-1}$ at a frequency of $8 \mathrm{~Hz}$. In contrast, in terms of the intrinsic attenuation, $Q_{\text {int }}^{-1}$ shows a stationary decay rate $\left(\propto f^{-0.7}\right)$ over a frequency range of $1-32 \mathrm{~Hz}$.

\section{Discussion}

\subsection{Seismic wave attenuation mechanism}

Our results indicate that, in the upper lithosphere beneath the Kanto region, scattering is the predominant attenuation mechanism at frequencies $<2 \mathrm{~Hz}$, whereas intrinsic attenuation dominates scattering attenuation at high frequencies. The transition of the predominant attenuation mechanism at a similar frequency has been reported in studies undertaken around the world (see a review by Yoshimoto and Jin, 2008). For example, Matsunami and Nakamura (2004) reported their consistent observation that the intrinsic absorption becomes predominant $>4 \mathrm{~Hz}$ on the basis of an envelope analysis of shallow crustal earthquakes in Wakayama, southwestern Japan. Mayeda et al. (1992) observed the transition of the predominant attenuation mechanism at $3 \mathrm{~Hz}$ by employing MLTWA for seismograms recorded in central California. In addition, there have been many studies on the apparent attenuation of $S$-waves that describe a similar change in the frequency dependence of $Q_{S}^{-1}$ at a few hertz (e.g., Ordaz and Singh, 1992; Horasan and Boztepe-Güney, 2004). Since the characteristic frequency at which the transition of the dominant attenuation mechanism occurs may exhibit regional variations that reflect regional tectonic activity, it is very interesting to investigate whether or not these $Q_{S}^{-1}$ observations can be interpreted in the same way.

In the present study, $Q_{S}^{-1}$ exhibits a weak frequency dependence at frequencies $>8 \mathrm{~Hz}$ in the Kanto region. This 

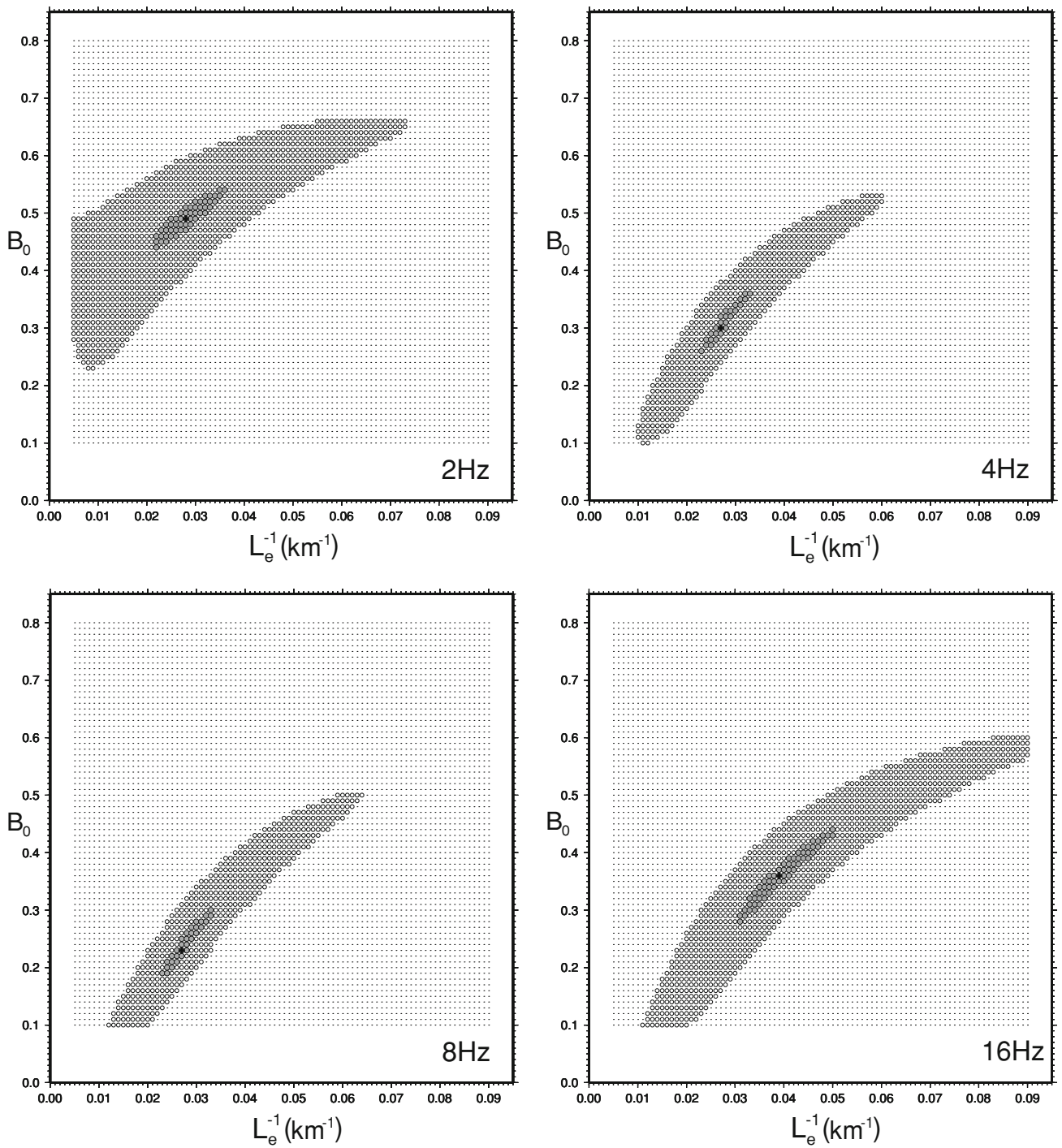

Fig. 4. Maps of the residuals for four frequency bands (center frequencies of 2, 4, 8, and $16 \mathrm{~Hz}$ ). The grid search was carried out at 6,106 points: $L_{\mathrm{e}}^{-1}=0.005,0.006,0.007, \cdots, 0.090 \mathrm{~km}^{-1}$ and $B_{0}=0.10,0.11,0.12, \cdots, 0.80$. The pair $L_{\mathrm{e}}^{-1}$ and $B_{0}$ of the minimum residual is indicated by the solid circle. Shaded circles indicate the approximate $68 \%$ confidence regions of these parameters. Open circles indicate the regions in which the residuals are less than 1.5 times the minimum residual.

characteristic can be interpreted in terms of the effect of the almost frequency independent $Q_{\text {scat }}^{-1}$ values at high frequencies. Adams and Abercrombie (1998) observed a similar change in the frequency dependence of $Q_{S}^{-1}$ at $10 \mathrm{~Hz}$ by employing MLTWA for seismograms recorded in the Cajon Pass borehole in Southern California. They reported a strong frequency dependence $\left(\propto f^{-1.8}\right)$ below $10 \mathrm{~Hz}$ and a weak frequency dependence $\left(\propto f^{-0.34}\right)$ at higher frequencies. Matsuzawa et al. (2003) observed a similar frequency dependence for $Q_{S}^{-1}$ using a twofold spectral ratio method for shallow crustal earthquakes that occurred in central Honshu, Japan. These results obtained in different regions suggest that the $Q_{\text {scat }}^{-1}$ value for the upper lithosphere tends to be almost frequency independent at high frequencies.

\subsection{Lithosphere heterogeneities}

Heterogeneities in the lithosphere have been stochastically investigated using a variety of seismic phenomena, such as the scattering attenuation of direct $S$-waves (Aki, 1980b; Sato, 1984), coda wave excitation (Wu and Aki, 1985b; Leary and Abercrombie, 1994), the broadening of seismogram envelopes (Sato, 1989; Gusev and Abubakirov, 1996; Saito et al., 2005), and the amplitude and phase correlation of teleseismic $P$-waves (Aki, 1973; Flatté and $\mathrm{Wu}, 1988)$. In these studies, Gaussian, exponential, von Kármán, and self-similar autocorrelation functions were adopted for characterizing the spatial variation in the fractional fluctuation of elastic parameters (e.g., $S$-wave velocity). In this case, the power spectral density of the heterogeneities in the lithosphere can be modeled using just two or three parameters.

It is well known that the scattering attenuation, $Q_{\text {scat }}^{-1}$, is a function of the power spectral density of the heterogeneities in the lithosphere (e.g., Frankel and Clayton, 1986). All stochastic models mentioned above, with the exception of the self-similar heterogeneities, predict a single peak for $Q_{\text {scat }}^{-1}$ with smooth amplitude decay on either side of it. In contrast, our observation reveals a rather complex frequency dependence, indicating multiple scale heterogeneities in the lithosphere (Wu and Aki, 1985a). 
Table 1. Estimation of the inverse of the extinction length $\left(L_{\mathrm{e}}^{-1}\right)$, seismic albedo $\left(B_{0}\right)$, apparent attenuation $\left(Q_{S}^{-1}\right)$, scattering attenuation $\left(Q_{\mathrm{scat}}^{-1}\right)$, and intrinsic attenuation $\left(Q_{\text {int }}^{-1}\right)$.

\begin{tabular}{|c|c|c|c|c|c|c|c|c|c|c|}
\hline \multirow{2}{*}{$\begin{array}{l}\text { Frequency }(\mathrm{Hz}) \\
1.000-1.414\end{array}$} & \multicolumn{2}{|c|}{$L_{\mathrm{e}}^{-1}\left(\mathrm{~km}^{-1}\right)$} & \multicolumn{2}{|c|}{$B_{0}$} & \multicolumn{2}{|c|}{$Q_{S}^{-1} \times 10^{-3}$} & \multicolumn{2}{|c|}{$Q_{\text {scat }}^{-1} \times 10^{-3}$} & \multicolumn{2}{|c|}{$Q_{\text {int }}^{-1} \times 10^{-3}$} \\
\hline & 0.026 & +0.011 & 0.63 & +0.04 & 13.92 & +5.89 & 8.77 & +3.75 & 5.15 & +2.25 \\
\hline & & -0.009 & & -0.04 & & -4.82 & & -3.09 & & -1.87 \\
\hline \multirow[t]{2}{*}{$1.189-1.682$} & 0.028 & +0.010 & 0.58 & +0.04 & 12.61 & +4.50 & 7.31 & +2.69 & 5.30 & +1.99 \\
\hline & & -0.009 & & -0.05 & & -4.05 & & -2.40 & & -1.78 \\
\hline \multirow[t]{2}{*}{$1.414-2.000$} & 0.026 & +0.009 & 0.54 & +0.05 & 9.84 & +3.41 & 5.31 & +1.90 & 4.53 & +1.64 \\
\hline & & -0.007 & & -0.05 & & -2.65 & & -1.51 & & -1.31 \\
\hline \multirow[t]{2}{*}{$1.682-2.378$} & 0.028 & +0.008 & 0.49 & +0.05 & 8.91 & +2.55 & 4.37 & +1.33 & 4.55 & +1.37 \\
\hline & & -0.006 & & -0.05 & & -1.91 & & -1.04 & & -1.07 \\
\hline \multirow[t]{2}{*}{$2.000-2.828$} & 0.029 & +0.007 & 0.44 & +0.05 & 7.76 & +1.87 & 3.42 & +0.91 & 4.35 & +1.12 \\
\hline & & -0.005 & & -0.05 & & -1.34 & & -0.71 & & -0.84 \\
\hline \multirow[t]{2}{*}{$2.378-3.364$} & 0.025 & +0.006 & 0.37 & +0.05 & 5.63 & +1.35 & 2.08 & +0.57 & 3.55 & +0.90 \\
\hline & & -0.004 & & -0.05 & & -0.90 & & -0.44 & & -0.63 \\
\hline \multirow[t]{2}{*}{$2.828-4.000$} & 0.025 & +0.006 & 0.33 & +0.05 & 4.73 & +1.14 & 1.56 & +0.47 & 3.17 & +0.81 \\
\hline & & -0.005 & & -0.06 & & -0.95 & & -0.39 & & -0.68 \\
\hline \multirow[t]{2}{*}{$3.364-4.757$} & 0.027 & +0.006 & 0.30 & +0.06 & 4.30 & +0.96 & 1.29 & +0.39 & 3.01 & +0.72 \\
\hline & & -0.004 & & -0.04 & & -0.64 & & -0.26 & & -0.48 \\
\hline \multirow[t]{2}{*}{$4.000-5.657$} & 0.029 & +0.005 & 0.32 & +0.05 & 3.88 & +0.67 & 1.24 & +0.29 & 2.64 & +0.50 \\
\hline & & -0.005 & & -0.05 & & -0.67 & & -0.29 & & -0.50 \\
\hline \multirow[t]{2}{*}{$4.757-6.727$} & 0.028 & +0.005 & 0.28 & +0.05 & 3.15 & +0.56 & 0.88 & +0.22 & 2.27 & +0.44 \\
\hline & & -0.004 & & -0.05 & & -0.45 & & -0.20 & & -0.36 \\
\hline \multirow[t]{2}{*}{$5.657-8.000$} & 0.027 & +0.006 & 0.24 & +0.06 & 2.56 & +0.57 & 0.61 & +0.21 & 1.94 & +0.46 \\
\hline & & -0.004 & & -0.05 & & -0.38 & & -0.16 & & -0.32 \\
\hline \multirow[t]{2}{*}{$6.727-9.514$} & 0.027 & +0.006 & 0.23 & +0.07 & 2.15 & +0.48 & 0.49 & +0.19 & 1.65 & +0.40 \\
\hline & & -0.004 & & -0.04 & & -0.32 & & -0.11 & & -0.26 \\
\hline \multirow[t]{2}{*}{$8.000-11.314$} & 0.032 & +0.006 & 0.28 & +0.05 & 2.14 & +0.40 & 0.60 & +0.19 & 1.54 & +0.33 \\
\hline & & -0.006 & & -0.07 & & -0.40 & & -0.16 & & -0.31 \\
\hline \multirow[t]{2}{*}{$9.514-13.454$} & 0.032 & +0.007 & 0.28 & +0.07 & 1.80 & +0.39 & 0.50 & +0.17 & 1.30 & +0.31 \\
\hline & & -0.006 & & -0.06 & & -0.34 & & -0.14 & & -0.27 \\
\hline \multirow[t]{2}{*}{$11.314-16.000$} & 0.035 & +0.009 & 0.32 & +0.07 & 1.66 & +0.43 & 0.53 & +0.19 & 1.13 & +0.32 \\
\hline & & -0.007 & & -0.08 & & -0.33 & & -0.16 & & -0.25 \\
\hline \multirow[t]{2}{*}{$13.454-19.027$} & 0.039 & +0.011 & 0.36 & +0.08 & 1.55 & +0.44 & 0.56 & +0.20 & 0.99 & +0.31 \\
\hline & & -0.008 & & -0.08 & & -0.32 & & -0.17 & & -0.24 \\
\hline \multirow[t]{2}{*}{$16.000-22.627$} & 0.045 & +0.012 & 0.41 & +0.07 & 1.51 & +0.40 & 0.62 & +0.21 & 0.89 & +0.27 \\
\hline & & -0.011 & & -0.09 & & -0.37 & & -0.18 & & -0.24 \\
\hline \multirow[t]{2}{*}{$19.027-26.909$} & 0.050 & +0.015 & 0.46 & +0.07 & 1.41 & +0.42 & 0.65 & +0.23 & 0.76 & +0.26 \\
\hline & & -0.013 & & -0.09 & & -0.37 & & -0.20 & & -0.22 \\
\hline \multirow[t]{2}{*}{$22.627-32.000$} & 0.054 & +0.016 & 0.50 & +0.06 & 1.28 & +0.38 & 0.64 & +0.22 & 0.64 & +0.22 \\
\hline & & -0.015 & & -0.09 & & -0.36 & & -0.19 & & -0.19 \\
\hline
\end{tabular}

In our study, to characterize the power spectral density of the heterogeneities in the upper lithosphere beneath the Kanto region, we combine the Gaussian and self-similar heterogeneities to explain the frequency dependence of $Q_{\text {scat }}^{-1}$ (Fig. 7). For this analysis, we adopt the Born approximation for evaluating the seismic scattering in random elastic media (Sato and Fehler, 1998). When we consider our observation $Q_{S}^{-1} \approx Q_{\text {scat }}^{-1}$ at low frequencies and the $Q_{S}^{-1}$ peak at $0.8 \mathrm{~Hz}$ observed by Kinoshita (1994) and reconfirmed by Kinoshita and Ohike (2002), the $Q_{\text {scat }}^{-1}$ value at frequencies between about 0.3 and $3 \mathrm{~Hz}$ may be approximately explained by the scattering caused by the Gaussian heterogeneities with a fractional fluctuation $\varepsilon \approx 10 \%$ and a correlation length $a \approx 2 \mathrm{~km}$. This fractional fluctuation value is of the same order as the perturbation of the $S$-wave velocity deduced from seismic tomography (e.g., Matsubara et al., 2005). The von Kármán heterogeneities with a high order $\kappa \approx 1$ may be another possible random structure model. However, such a model would fail to explain the steep fall-off observed for $Q_{\text {scat }}^{-1}$ except when its peak is at a much lower frequency than that found by Kinoshita (1994).

For the frequency-independent $Q_{\text {scat }}^{-1}$ at high frequencies, self-similar heterogeneities with $\varepsilon=1-2 \%$ and $a>0.5 \mathrm{~km}$ might induce scattering attenuation. Since the uncertainty in the cut-off scattering angle, which is required in the $Q_{\text {scat }}^{-1}$ estimation (Sato and Fehler, 1998), prevents us from estimating $\varepsilon$ precisely from the observed $Q_{\text {scat }}^{-1}$ (see figure 8 of Frankel and Clayton, 1986), we roughly approximated $\varepsilon$ in our analysis.

The interpretation above implies that the upper lithosphere is rich in short-wavelength heterogeneities, which coincides with the borehole observations in the Kanto region reported by Shiomi et al. (1997) and shows self-similar heterogeneous properties. It is interesting that although 


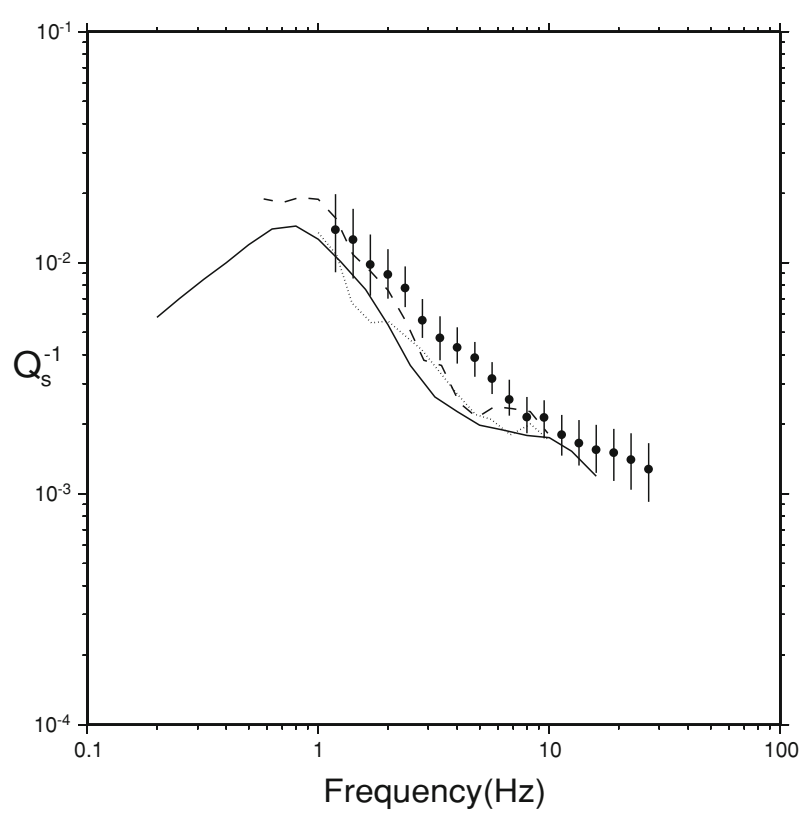

Fig. 5. Frequency dependence of $Q_{S}^{-1}$ measured in and around the Kanto region. Our estimation in this study is indicated by the solid circle with the error bar (68\% confidence limit). The comparative plot shows the $Q_{S}^{-1}$ values obtained by Takemura et al. (1991) (dotted line), Kato et al. (1998) (broken line), and Kinoshita and Ohike (2002) (solid line).

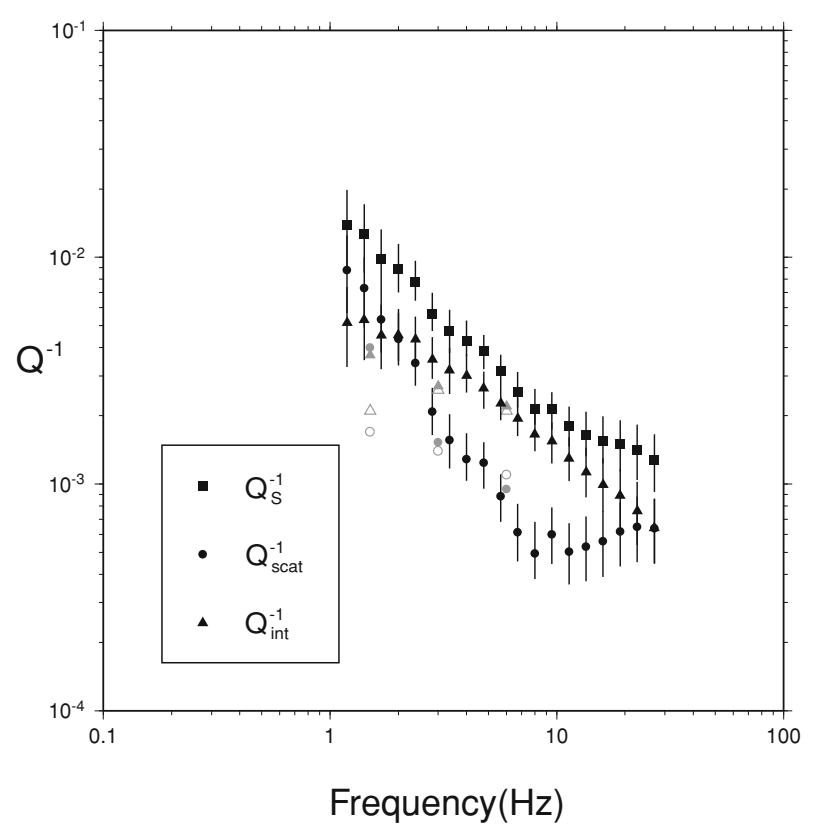

Fig. 6. Frequency dependence of $Q_{S}^{-1}, Q_{\text {scat }}^{-1}$, and $Q_{\text {int }}^{-1}$. The comparative plot shows the $Q_{\text {scat }}^{-1}$ and $Q_{\text {int }}^{-1}$ values obtained by Fehler et al. (1992) (open gray) and Hoshiba (1993) (filled gray).

Adams and Abercrombie (1998) did not estimate $\varepsilon$, they also adopted the Gaussian and self-similar models to characterize the heterogeneities in the upper lithosphere in their analysis of $S$-wave attenuation in Southern California. It can be speculated that a different geophysical process had formed the Gaussian and self-similar heterogeneities. However, this remains an open question and includes a point of particular interest, namely whether there are other large characteristic scale lengths as reported in analyses of the

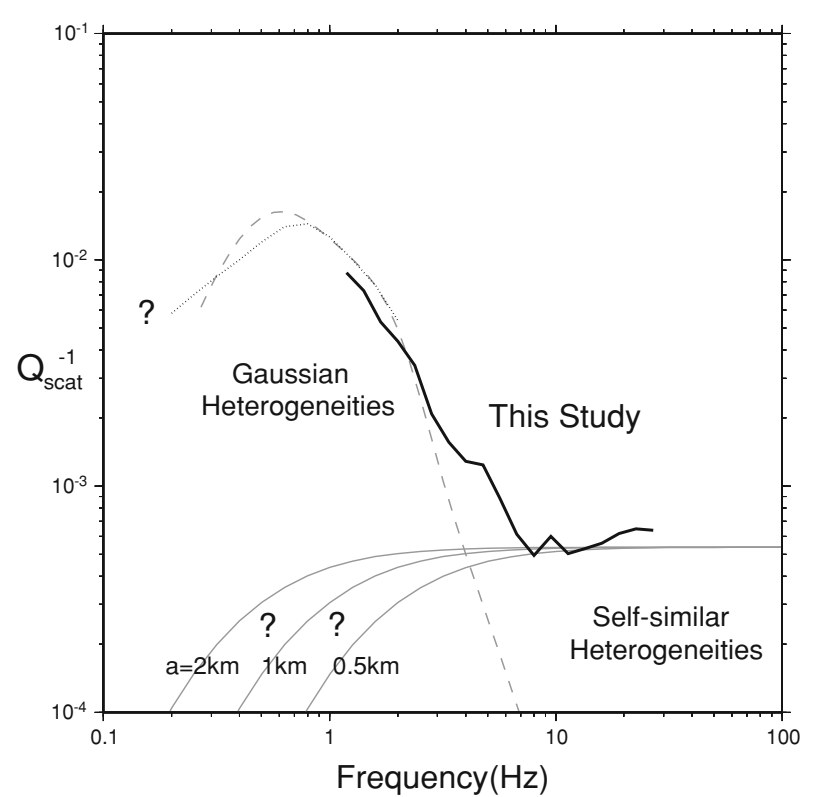

Fig. 7. An interpretation of the relationship between the scattering attenuation and the heterogeneities in the lithosphere. The frequency dependence of $Q_{\text {scat }}^{-1}$ estimated in this study is indicated by a solid line. $Q_{S}^{-1}$ measured by Kinoshita and Ohike (2002) for frequencies $<2 \mathrm{~Hz}$ is shown by a dotted line. Theoretical $Q_{\text {scat }}^{-1}$ curves are calculated for the Gaussian heterogeneities (gray broken line: $\varepsilon=10 \%, a=2 \mathrm{~km}$ ) and the self-similar heterogeneities (gray line: $\varepsilon=1.4 \%, a=0.5,1$, and $2 \mathrm{~km}$ ), where a cut-off scattering angle of $29^{\circ}$ is assumed.

amplitude and phase fluctuation of teleseismic $P$-waves (e.g., Aki, 1973).

\subsection{Coda excitation}

The discussion above on the scattering attenuation indicates that the random heterogeneities characterized by the Gaussian autocorrelation function with a fractional fluctuation $\varepsilon \approx 10 \%$ and a correlation length $a \approx 2 \mathrm{~km}$ in the upper lithosphere beneath the Kanto region are superimposed on the weak background self-similar heterogeneity. It is surprising that the magnitude of the background fluctuation $(\varepsilon=1-2 \%)$ is comparable to the velocity fluctuations in the upper lithosphere in tectonically stable regions, such as the Baltic Shield in northern Europe (Line et al., 1998). Is this randomness sufficiently strong to excite coda waves for local earthquakes?

To answer this question, we theoretically estimated the $S$-to- $S$ backscattering coefficient $g_{\pi}^{S S}$ for the background self-similar fluctuation. Using equation (4.54) of Sato and Fehler (1998), $g_{\pi}^{S S} \approx 2.54 \varepsilon^{2} l$ is obtained for al $\gg 0$, where $l=2 \pi f / V_{S}$. From this relation, we found that the value of $g_{\pi}^{S S}$ at a frequency of $10 \mathrm{~Hz}$ is about $1 \times$ $10^{-2} \mathrm{~km}^{-1}$, which falls within the value range reported in previous studies on coda wave excitation (Sato and Fehler, 1998). This indicates that the weak background self-similar heterogeneities have the potential to excite seismic coda waves at frequencies above $10 \mathrm{~Hz}$.

It is certain that the upper lithosphere has a more complex structure than that assumed in this paper. For example, the characteristics of heterogeneities may change with depth and horizontal location as reported in seismic tomography studies. Moreover, heterogeneities may be 
non-isotropic and show a preferred orientation of continuity (e.g., Furumura and Kennett, 2005). However, we believe that our present attempts may be the first step towards a good understanding of the frequency dependence and attenuation mechanism of $S$-waves in the upper lithosphere.

\section{Conclusion}

The frequency dependence of the apparent, scattering, and intrinsic $S$-wave attenuations of the upper lithosphere was measured in the $1-$ to $32-\mathrm{Hz}$ frequency range in the Kanto region using MLTWA. A new set of time windows was proposed for MLTWA in which multiple isotropic scattering was assumed. From an analysis of 115 borehole seismograms of local earthquakes, we obtained a highfrequency resolution for $Q_{S}^{-1}$, which agrees well with the results of previous studies undertaken in and around this region. The MLTWA revealed that scattering attenuation dominates intrinsic attenuation in the attenuation mechanism at low frequencies $(<2 \mathrm{~Hz})$, whereas intrinsic attenuation is the predominant attenuation mechanism at high frequencies. This transition, which results from the different frequency dependence of $Q_{\text {scat }}^{-1}\left(\propto f^{-1.5}\right)$ and $Q_{\text {int }}^{-1}\left(\propto f^{-0.7}\right)$ at this frequency, can be seen in the frequency dependence of $Q_{S}^{-1}$. Interestingly, $Q_{\text {scat }}^{-1}$ is almost frequency independent at frequencies $>8 \mathrm{~Hz}$, suggesting the self-similar nature of the short-wavelength heterogeneities in the upper lithosphere of the Kanto region. The $Q_{\text {scat }}^{-1}$ frequency dependence obtained in this study and $Q_{S}^{-1}$ reported by Kinoshita (1994) indicate that the random heterogeneities characterized by the Gaussian autocorrelation function with a fractional fluctuation $\varepsilon \approx 10 \%$ and a correlation length $a \approx$ $2 \mathrm{~km}$ in the upper lithosphere are superimposed on weak background self-similar heterogeneities.

Acknowledgments. We used borehole data from the Hi-net provided by the National Research Institute for Earth Science and Disaster Prevention and hypocentral information provided by the Japan Meteorological Agency. We thank Y. Asano and N. Tsumura for constructive review of the manuscript. We are grateful to S. Kinoshita for his encouragement and discussions. Special thanks should also go to M. Kamoshita and M. Ohsawa for their contribution to this study. Most of the figures were created using GMT (Wessel and Smith, 1995). This study was supported by the Grant-in-Aid for Scientific Research (C) No. 20540413 of the Japan Society for the Promotion of Science (JSPS).

\section{References}

Abubakirov, I. R., Attenuation characteristics of transverse waves in the lithosphere of Kamchatka estimated from observations at the Petropavlovsk digital broadband station, Phys. Solid Earth, 41, 813824,2005

Adams, D. A. and R. E. Abercrombie, Seismic attenuation above $10 \mathrm{~Hz}$ in southern California from coda waves recorded in the Cajon Pass borehole, J. Geophys. Res., 103, 24257-24270, 1998.

Aki, K., Scattering of P waves under the Montana Lasa, J. Geophys. Res., 78, 1334-1346, 1973.

Aki, K., Attenuation of shear-waves in the lithosphere for frequencies from 0.05 to $25 \mathrm{~Hz}$, Phys. Earth Planet. Inter, 21, 50-60, 1980a.

Aki, K., Scattering and attenuation of shear waves in the lithosphere, $J$. Geophys. Res., 85, 6496-6504, 1980b.

Felher, M., M. Hoshiba, H. Sato, and K. Obara, Separation of scattering and intrinsic attenuation for the Kanto-Tokai region, Japan, using measurements of $S$-wave energy versus hypocentral distance, Geophys. $J$. Int., 108, 787-800, 1992

Flatté, S. M. and R. S. Wu, Small-scale structure in the lithosphere and asthenosphere deduced from arrival time and amplitude fluctuations at
NORSAR, J. Geophys. Res., 93, 6601-6614, 1988.

Frankel, A. and R. W. Clayton, Finite difference simulations of seismic scattering: implications for the propagation of short-period seismic waves in the crust and models of crust heterogeneity, J. Geophys. Res., 91, 6465-6489, 1986.

Furumura, T. and B. L. N. Kennett, Subduction zone guided waves and the heterogeneity structure of the subducted plate: Intensity anomalies in northern Japan, J. Geophys. Res., 110, B10302, doi:10. 1029/2004JB003486, 2005.

Gusev, A. A. and I. R. Abubakirov, Simulated envelopes of nonisotropically scattered body waves as compared to observed ones: another manifestation of fractal heterogeneity, Geophys. J. Int., 127, 4960, 1996.

Horasan, G. and A. Boztepe-Güney, S-wave attenuation in the Sea of Marmara, Turkey, Phys. Earth Planet. Inter., 142, 215-224, 2004.

Hoshiba, M., Separation of scattering attenuation and intrinsic absorption in Japan using the multiple lapse time window analysis form full seismogram envelope, J. Geophys. Res., 98, 15809-15824, 1993.

Hoshiba, M., Estimation of nonisotropic scattering in western Japan using coda wave envelopes: Application of a multiple nonisotropic scattering model, J. Geophys. Res., 100, 645-657, 1995.

Hoshiba, M., Large fluctuation of wave amplitude produced by small fluctuation of velocity structure, Phys. Earth Planet. Inter., 120, 201-217, 2000

Kato, K., M. Takemura, and K. Yashiro, Regional variation of source spectra in high-frequency range determined from strong motion records, Zisin 2, 51, 123-138, 1998 (in Japanese with English abstract).

Kinoshita, S., Frequency-dependent attenuation of shear waves in the crust of the Southern Kanto area, Japan, Bull. Seismol. Soc. Am., 84, 13871396, 1994.

Kinoshita, S. and M. Ohike, Scaling relations of earthquakes that occurred in the upper part of the Philippine Sea Plate beneath the Kanto region, Japan, estimated by means of borehole recordings, Bull. Seismol. Soc. Am., 92, 611-624, 2002.

Leary, P. and R. Abercrombie, Fractal fracture scattering origin of S-wave coda: spectral evidence from recordings at $2.5 \mathrm{~km}$, Geophys. Res. Lett., 21, 1683-1686, 1994.

Line, C. E. R., R. W. Hobbs, J. A. Hudson, and D. B. Snyder, Statistical inversion of controlled-source seismic data using parabolic wave scattering theory, Geophys. J. Int., 132, 61-78, 1998.

Matsubara, M., H. Hayashi, K. Obara, and K. Kasahara, Low-velocity oceanic crust at the top of the Philippine Sea and Pacific plates beneath the Kanto region, central Japan, imaged by seismic tomography, J. Geophys. Res., 110, B12304, doi: 10.1029/2005JB003673, 2005.

Matsunami, K. and M. Nakamura, Seismic attenuation in a nonvolcanic swarm region beneath Wakayama, southwest Japan, J. Geophys. Res., 109, B09302, doi: 10.1029/2003JB002758, 2004.

Matsuzawa, T., M. Takeo, S. Ide, Y. Iio, H. Ito, K. Imanishi, and S. Horiuchi, Estimation of the S-wave attenuation in the western Nagano region from twofold spectral ratio, Zisin 2, 56, 75-88, 2003 (in Japanese with English abstract).

Mayeda, K., S. Koyanagi, M. Hoshiba, K. Aki, and Y. Zeng, A comparative study of scattering, intrinsic and coda $Q^{-1}$ for Hawaii, Long Valley, and central California between 1.5 and $15.0 \mathrm{~Hz}$, J. Geophys. Res., 97, 6643$6659,1992$.

Nakamura, R., K. Satake, S. Toda, T. Uetake, and S. Kamiya, Threedimensional attenuation $(Q s)$ structure beneath the Kanto district, Japan, as inferred from strong motion records, Geophys. Res. Lett., 33, L21304, doi:10.1029/2006GL027352, 2006.

Okada, Y., K. Kasahara, S. Hori, K. Obara, S. Sekiguchi, H. Fujiwara, and A. Yamamoto, Recent progress of seismic observation networks in Japan-Hi-net, F-net, K-NET and KiK-net-, Earth Planets Space, 56, xv-xviii, 2004

Ordaz, M. and S. K. Singh, Source spectra and spectral attenuation of seismic waves from Mexican earthquakes, and evidence of amplification in the hill zone of Mexico City, Bull. Seismol. Soc. Am., 82, 24-43, 1992.

Saito, T., H. Sato, M. Ohtake, and K. Obara, Unified explanation of envelope broadening and maximum-amplitude decay of high-frequency seismograms based on the envelope simulation using the Markov approximation: forearc side of the volcanic front in northeastern Honshu, Japan, J. Geophys. Res., 110, B01304, doi:10.1029/2004JB003225, 2005.

Sato, H., Attenuation and envelope formation of three-component seismograms of small local earthquakes in randomly inhomogeneous lithosphere, J. Geophys. Res., 89, 1221-1241, 1984.

Sato, H., Broadening of seismogram envelopes in the randomly inhomogeneous lithosphere based on the parabolic approximation: southeastern 
Honshu, Japan, J. Geophys. Res., 94, 17735-17747, 1989.

Sato, H. and M. C. Fehler, Seismic Wave Propagation and Scattering in the Heterogeneous Earth, Springer-Verlag New York, 1998.

Shiomi, K., H. Sato, and M. Ohtake, Broad-band power-law spectra of well-log data in Japan, Geophys. J. Int., 130, 57-64, 1997.

Takemura, M., K. Kato, T. Ikeura, and E. Shima, Site amplification of $S$ waves from strong motion records in special relation to surface geology, J. Phys. Earth, 39, 537-552, 1991.

Tsumura, K., Determination of earthquake magnitude from duration of oscillation, Zisin 2, 20, 30-40, 1967 (in Japanese with English abstract).

Ueno, H., S. Hatekeyama, T. Aketagawa, J. Funasaki, and N. Hamada, Improvement of hypocenter determination procedures in the Japan Meteorological Agency, Quart. J. Seismol., 65, 123-134, 2002 (in Japanese with English abstract).

Wessel, P. and W. H. F. Smith, New version of the generic mapping tools released, Eos Trans. AGU, 76, 329, 1995.

$\mathrm{Wu}, \mathrm{R}$. S. and K. Aki, Elastic wave scattering by a random medium and the small-scale inhomogeneities in the lithosphere, J. Geophys. Res., 90,
10261-10273, 1985a.

$\mathrm{Wu}, \mathrm{R}$. S. and K. Aki, The fractal nature of the inhomogeneities in the lithosphere evidenced from seismic wave scattering, Pure Appl. Geophys., 123, 805-818, 1985b.

Yoshimoto, K. and A. Jin, Coda energy distribution and attenuation, in Earth Heterogeneity and Scattering Effects on Seismic Waves, edited by R. Dmowska, H Sato, and M. Fehler, Advances in Geophysics, Vol. 50, 265-299, Elsevier, 2008.

Yoshimoto, K., H. Sato, and M. Ohtake, Frequency-dependent attenuation of $P$ and $S$ waves in the Kanto area, Japan, based on the codanormalization method, Geophys. J. Int., 114, 165-174, 1993.

Zeng, Y., F. Su, and K. Aki, Scattering wave energy propagation in a random isotropic scattering medium: 1. Theory, J. Geophys. Res., 96, 607-619, 1991.

K. Yoshimoto (e-mail: k_yoshi@yokohama-cu.ac.jp) and M. Okada 